\title{
Integrated analysis of differentially expressed genes and construction of a competing endogenous RNA network in human Huntington neural progenitor cells
}

\author{
Xiaoping Tan, Yang Liu, Taiming Zhang and Shuyan Cong ${ }^{*}$ (])
}

\begin{abstract}
Background: Huntington's disease (HD) is one of the most common polyglutamine disorders, leading to progressive dyskinesia, cognitive impairment, and neuropsychological problems. Besides the dysregulation of many proteincoding genes in HD, previous studies have revealed a variety of non-coding RNAs that are also dysregulated in HD, including several long non-coding RNAs (IncRNAs). However, an integrated analysis of differentially expressed (DE) genes based on a competing endogenous RNA (ceRNA) network is still currently lacking.

Methods: In this study, we have systematically analyzed the gene expression profile data of neural progenitor cells (NPCs) derived from patients with HD and controls (healthy controls and the isogenic controls of HD patient cell lines corrected using a CRISPR-Cas9 approach at the HTT locus) to screen out DE mRNAs and DE IncRNAs and create a ceRNA network. To learn more about the possible functions of IncRNAs in the ceRNA regulatory network in HD, we conducted a functional analysis of Gene Ontology (GO) and Kyoto Encyclopedia of Genes and Genomes (KEGG) and established a protein-protein interaction (PPI) network for mRNAs interacting with these IncRNAs.

Results: We identified 490 DE mRNAs and 94 DE IncRNAs, respectively. Of these, 189 mRNAs and 20 IncRNAs were applied to create a ceRNA network. The results showed that the function of DE IncRNAs mainly correlated with transcriptional regulation as demonstrated by GO analysis. Also, KEGG enrichment analysis showed these IncRNAs were involved in tumor necrosis factor, calcium, Wnt, and NF-kappa B signaling pathways. Interestingly, the PPI network revealed that a variety of transcription factors in the ceRNA network interacted with each other, suggesting such IncRNAs may regulate transcription in HD by controlling the expression of such protein-coding genes, especially transcription factors.
\end{abstract}

Conclusions: Our research provides new clues for uncovering the mechanisms of IncRNAs in HD and can be used as the focus for further investigation.

Keywords: RNA, IncRNA, Huntington disease, ceRNA, Bioinformatics

*Correspondence: whenshan524@163.com

Department of Neurology, Shengjing Hospital of China Medical

University, Shenyang, 36 Sanhao Street, Shenyang 110004, Liaoning,

People's Republic of China

\section{Background}

Huntington's disease (HD) is an autosomal dominantinherited polyglutamine disorder. Typical features include progressive movement disorder, neuropsychiatric problems, and cognitive impairment. Abnormal amplification of cytosine-adenine-guanine (CAG) repeats 
of the first exon in the huntingtin (HTT) gene encoding an abundantly-expressed 3144 amino acid protein, is the root cause of this fatal disease [1]. The DNA of healthy persons contains a region of less than 36 CAG repeats that encodes a polyglutamine (polyQ) tract in the huntingtin gene. An extension in the length of the polyQ tract at the $\mathrm{N}$-terminal of the mutant huntingtin protein (mutHTT), encoded by an abnormal HTT gene containing an expansion of CAG repeats (> 36 in length), changes the conformation of mutHTT and leads to intracellular protein aggregates [2]. Carriers with longer CAG repeats of $H T T$ exhibit different severities of HD, depending on the unusual length of CAG repeats [3]. How this pure mutant of the ubiquitously expressed protein leads to specific neurodegeneration is, however, unclear.

Over the past few decades, along with identifying the HTT gene, considerable progress has been made in learning the mechanism of neurodegeneration caused by mutHTT, including increased excitotoxicity injury in neurons through a deficiency of wild-type HTT [4], nutritional support disorders from neurons [5], the appearance of inclusion bodies derived from aberrant protein accumulation and the influence of polyglutamine length on the extent of mutHTT aggregates [6], mitochondrial impairment [7], and defective axonal transport in HD neurons $[8,9]$. In addition to such cellular dysfunctions, mutHTT also caused complex transcriptional and post-transcriptional changes in mouse models and the brains of patients with HD, particularly in the striatum, followed by the cortex and cerebellum; the severity of transcriptional changes reflected the seriousness of neurodegeneration $[10,11]$. Dysregulation of the RE1 silencing transcription factor (REST) [12] and its target gene regulatory networks, including microRNA (miRNAs) mir-132 [13], mir-9 [14], mir-124 [15] and possibly other non-coding RNAs (ncRNAs), was particularly attractive as an explanation of the neurodegeneration caused by mutHTT in related studies.

Recently, increasing studies have investigated the role of transcriptional and post-transcriptional dysregulation in the pathogenesis of HD, including a variety of both protein-coding and ncRNAs. In particular, the dysregulation of the protein-coding RNA, REST, in HD has become attractive. As a subtype of ncRNAs, long noncoding RNAs (lncRNAs) are a class of transcripts containing more than 200 bases that do not have a functional open reading frame, and play crucial biological roles in epigenetics and transcriptional regulation [16]. In addition to being involved in normal physiological processes, previous studies have shown that lncRNAs take part in the pathogenesis of HD by regulating the expression of protein-coding genes via both cis- and trans-action pathways [17]. They also interact with varying repressive chromatin regulatory complexes (PRC2, RCOR1, and SMCX), and affect transcription factor function $[18,19]$. MicroRNAs are endogenous ncRNAs, approximately 22 nucleotides in length, that are involved in the negative regulation of mRNA at the post-transcriptional level by targeting a repressive protein complex (RISC) and pairing to the 3 '-untranslated region of mRNA to directly repress post-transcriptional translation [20]. Increasing evidence has illustrated how several neuronal-specific miRNAs were dysregulated in HD disease [12], some of which were targeted by REST. In 2011, the competition endogenous RNA (ceRNA) hypothesis was proposed that emphasized how mRNAs, transcribed pseudogenes, and lncRNAs could interact with each other through competitive binding to miRNA response elements (MREs) [21]. It is therefore necessary to establish an IncRNAmiRNA-mRNA-ceRNA regulatory network in HD.

Increasing evidence has shown that lncRNAs, as well as ceRNAs, were associated with HD [17]. Clarifying the changes of the lncRNA regulatory network in HD neurons may perhaps help to identify and manage this intractable neurodegenerative disease. In this study, we acquired lncRNA and mRNA expression profile data of neural progenitor cells (NPCs) that were differentiated from HD (including corrected HD by a CRISPR-Cas9 approach) and healthy human-induced pluripotent stem cells (iPSCs) in the Gene Expression Omnibus (GEO) database. We then constructed a ceRNA regulatory network using differentially expressed genes (DEGs) that may help us to shed light on the mechanism of transcriptional changes in HD neurons.

\section{Methods}

\section{Data acquisition and pre-processing}

The expression dataset used in this study was collected from a previous study of NPC samples generated by HD human iPSCs acquired from the GEO database (https:// www.ncbi.nlm.nih.gov/geo/) on the NCBI website. Series Matrix Files of GEO Series Accession NO.GSE93767 and the corresponding platform file of GPL10558 using an Illumina HumanHT-12 v4 Expression BeadChip (Illumina Inc., San Diego, CA, USA) were downloaded. This experiment was designed to explore transcriptional differences between HD and control cell lines, both of them derived from the HD (CAG180) / healthy (CAG33) human iPSCs and hiPSC-derived NPCs (the parental cell types were derived from human fibroblasts), and to identify genome-wide molecular changes after correction of the mutation in the HD cell line using a CRISPR/ Cas9 and piggyBac transposon-based approach [22]. A Cas9 nickase (Cas9n)-mediated cleavage of CAG180 iPSCs was carried out using a pair of selected small-guide RNAs at the HTT locus to correct the disease mutation. 
A piggyBac transposon selection cassette-based homologous recombination donor was used to establish isogenic control lines. After genome editing and targeted clones screening, a whole-exome sequencing was performed that identified a low off-target activity in a comparison of the isogenic control iPSCs and their parental CAG180 line. All of these cell lines were then efficiently differentiated into forebrain NPCs [22]. In this paper, two corrected isogenic controls of CAG180 HD NPC cell lines (HD-C\#1,2), and the non-isogenic CAG33 healthy NPC cell line, were included in the control group; the noncorrected CAG180 HD NPC cell line was included in the HD group. A total of 12 samples were used for this analysis, with three replicates per cell line per cell type. Expression values in the expression matrix file were the log-converted and adjusted data [22].

Due to the probes in the HumanHT-12 v4 Expression BeadChip derived from an earlier version of the NCBI Reference Sequence (RefSeq), RNAs Release 38, we rematched all 48,107 probe sequences to human NCBI RefSeq RNA Release 109 using a Basic Local Alignment Search Tool (optimized for highly similar sequences) from NCBI, 38,640 probes were retained. All probes were then converted to their corresponding gene symbols. Before differential expression analysis, if a gene symbol matched more than one probe, mean processing of the data was undertaken for all corresponding probes. We then analyzed these expression values using an R-Studio tool (version 1.1.463). All expression values were normalized by quantile normalization in package limma [23]. To discover which genes were targeting lncRNAs or proteincoding genes, we remapped these genes to the human reference genome annotation file downloaded from NCBI (Homo_sapiens.GRCh38.94.chr.gtf); genes without corresponding annotation information were removed. After the above filtering, 20,897 genes in the final dataset remained.

\section{Differentially expressed genes and construction of IncRNA-associated ceRNA network}

Differential expression analysis was performed in R studio software version 1.1.463 [24] using a Bioconductor 3.8 package (http://www.bioconductor.org/), with an absolute $\log _{2}$ fold-change $(|\operatorname{logFC}|)>1$ and $P<0.05$ as cutoff values by using an empirical Bayes method for screening DE lncRNAs and mRNAs. A lncRNA-related ceRNA regulatory network was constructed according to the "ceRNA hypothesis", in which lncRNA can regulate the expression of mRNAs by competitively binding miRNA, which contains common MREs. Therefore, the miRNAs targeted to DE lncRNAs were predicted by starBase v3.0 based on Ago CLIP-sequencing (seq) data with a threshold of high stringency $(\geq 3)[25]$ or by miRcode based on highly conserved miRNA targets [26]. Next, the DE mRNAs targeted-miRNAs were predicted by at least by two or more databases among miRDB [27], TargetScanHuman 7.2 [28], and the experimentally validated miRNA-target interactions database miRTarBase 7.0 [29]. Based on the acquired DE lncRNAs, DE mRNAs, and their co-targeted miRNAs, a ceRNA regulatory network was established and depicted by Cytoscape v3.7.0 software [30]. A flow diagram (Fig. 1) clearly shows how we undertook data analysis.

\section{PPI network analysis}

To better identify critical genes and clarify the potential relationships of mRNAs in the ceRNA network, protein-protein (PPI) network analysis was carried out using STRING software (score $>0.4$ ) [31].

\section{GO, KEGG functional enrichment analysis}

To clarify which biological processes and pathways of NPCs induce significant changes in HD for all aberrantly expressed protein-coding RNAs and those included in the ceRNA network, we performed functional enrichment analysis of GO using R software [32]. We created and visualized a functional enrichment network of KEGG pathways using Cytoscape plug-in ClueGO v2.5.4 [33] and CluePedia v1.5.4 [34] with the human genome as a background. The ClueGO network was built using kappa statistics to reflect the relationship between terms according to the resemblance of related genes. Functionally grouped networks with terms as nodes were connected based on their kappa score level $(\geq 0.4)$. Pathways showing a $p$-value $<0.05$ were regarded as remarkably enriched for both GO and KEGG analyses.

\section{Results}

\section{Probe re-annotation}

A total of 38,640 probes were re-annotated into corresponding gene symbols, and after deduplication using a mean method and data normalization, a final set of 2743 lncRNAs and 18,015 mRNAs was retained in the matrix file for subsequent analysis.

\section{Identification of differentially expressed genes}

Compared with the control group (CAG33 and HD-C\#1, 2), a total of 94 DE lncRNAs (49 up-regulated, 45 downregulated) and $490 \mathrm{DE}$ mRNAs (229 up-regulated, 261 down-regulated) were found in HD cell lines with 180 CAG repeats by differentially expression analysis. The strength of differential gene expression was shown in the form of volcano plots, and the top $20 \mathrm{up} /$ down-regulated DE lncRNAs and mRNAs were represented by heatmaps, respectively (Fig. 2). 


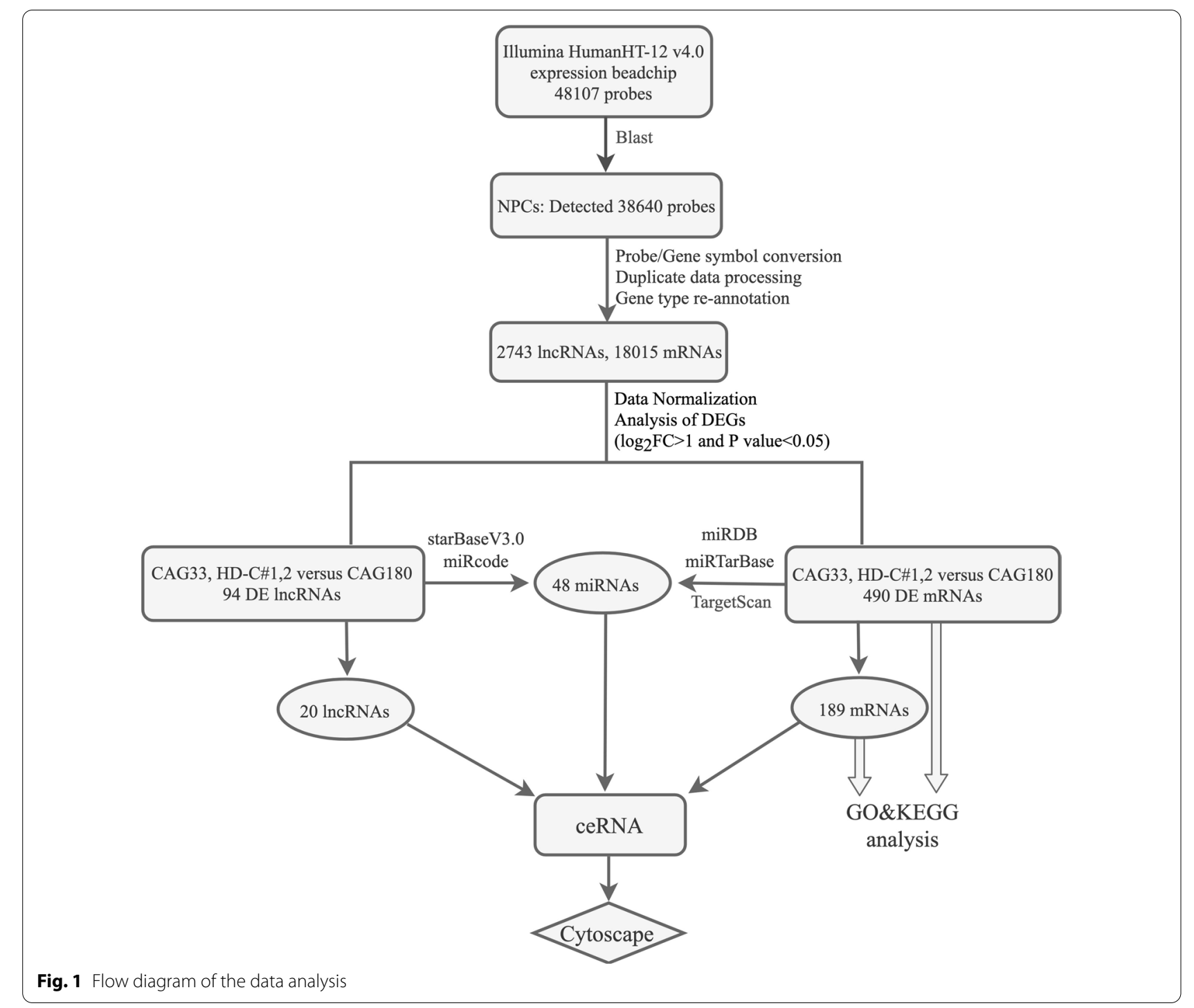

\section{Function analysis of DE mRNAs}

We further investigated the biological functions of upand down-regulated protein-coding mRNAs using Gene Ontology (GO) and Kyoto Encyclopedia of Genes and Genomes (KEGG) analyses. As shown in Fig. 3, the top 20 biological processes (BPs) and molecular functions (MFs) of protein-coding RNAs were exhibited as dot plots. We found that the significant changes in BPs of up-regulated mRNAs included the regulation of GTPase activity, a response to lipopolysaccharide, second messenger-mediated signaling, immune responses, spinal cord, and neurodevelopment and differentiation. For down-regulated mRNAs, their BPs included T-cell activation, the positive regulation of nervous system development and cell adhesion, the response to bone morphogenetic protein
(BMP), the immune response, $\mathrm{T}$ cell cytokine production, and neuron maturation. Similarly, the significant MF changes of up-regulated mRNAs included calmodulin binding, transcription factor activity, RNA polymerase II distal enhancer/proximal promoter sequence-specific DNA binding, iron ion binding, phospholipase $\mathrm{C}$ activity, and transmembrane transporter activity. Receptor ligand activity, channel activity, serine-type peptidase/hydrolase activity, and transcriptional activator activity were related to the MFs of down-regulated mRNAs.

To identify which pathways were involved in the DEGs, all DE mRNAs were matched to pathways in the KEGG database, and 34 significantly enriched pathways were identified ( $p<0.05$; Fig. 4$)$. When functionally grouped based on shared genes between terms with a kappa 


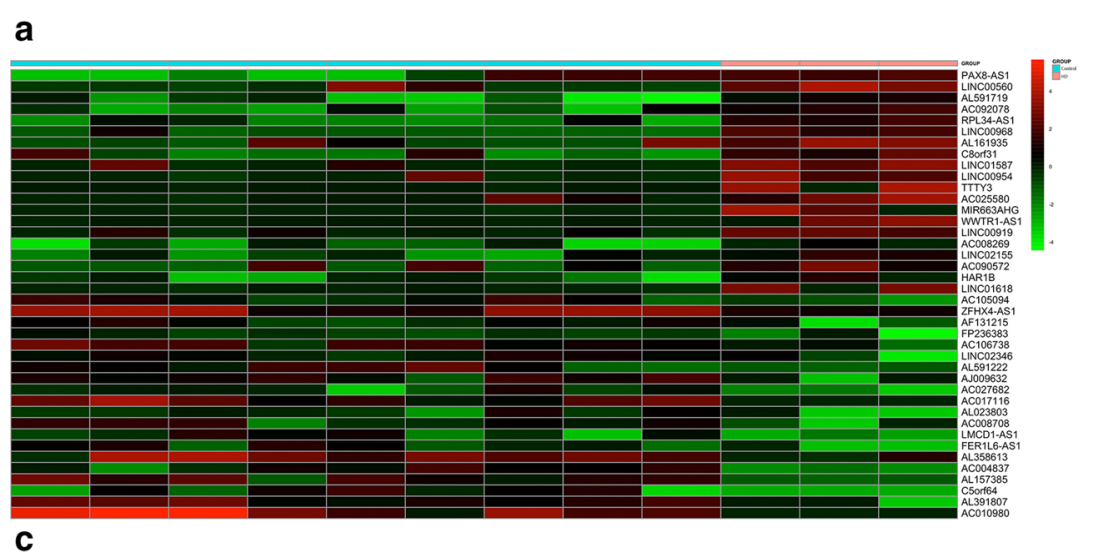

b
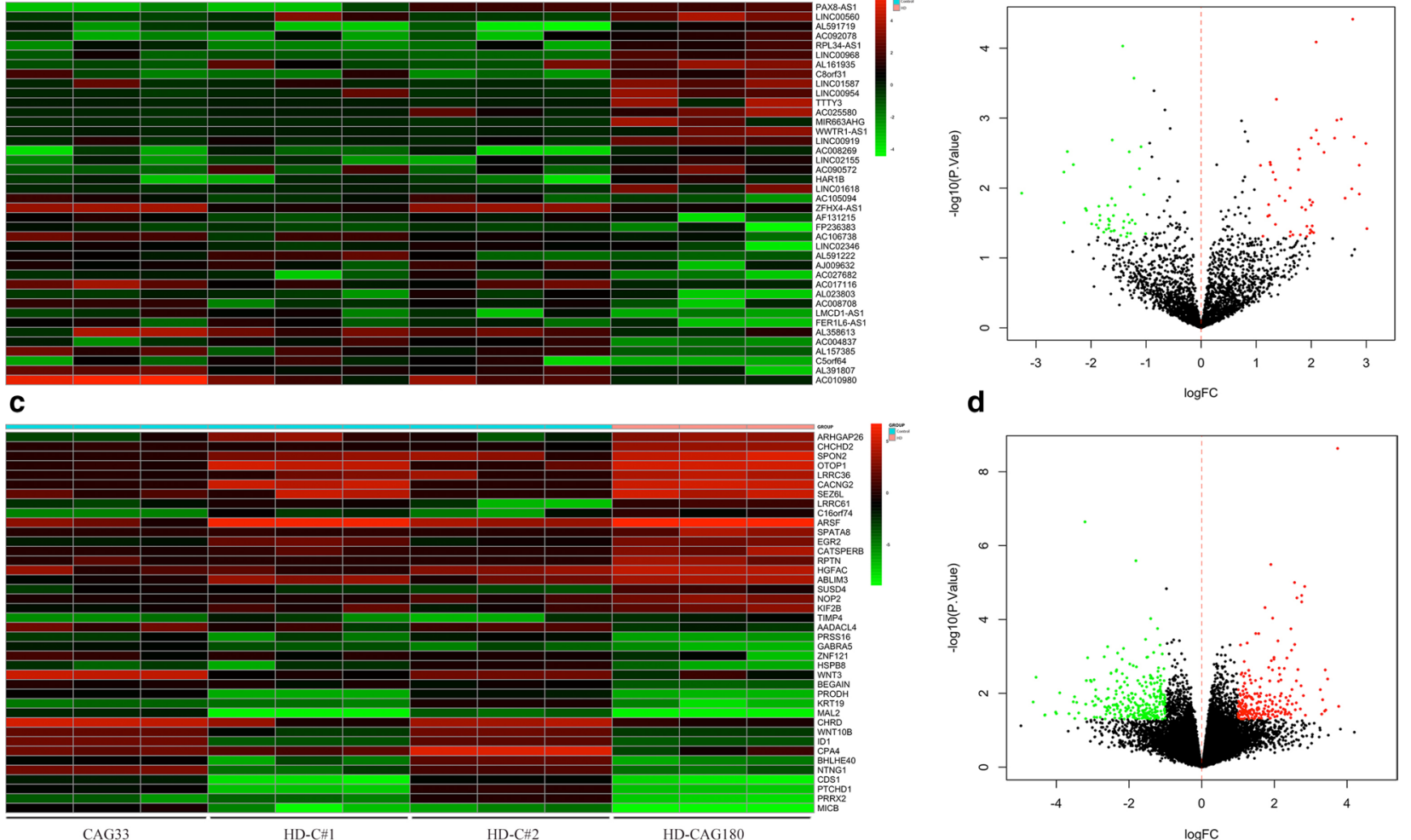

Fig. 2 DEGs analysis and visualization. a, c. Heat map of the top 20 differentially expressed (DE) long non-coding RNAs (IncRNAs) and mRNAs. b, d. Volcano plots of IncRNAs and mRNAs. Red and green represented up- and down-regulated differentially expressed genes (DEGs), respectively

score level $(\geq 0.4), 12$ functional groups were obtained in the final analysis, such as the calcium signaling pathway, tumor necrosis factor (TNF) signaling pathway, cell adhesion molecules, and NF-kappa B signaling pathway.

\section{LncRNA-associated ceRNA network}

As miRNA sponges, lncRNAs can isolate and bind miRNAs to regulate mRNA expression. By using the predictive databases of starBase, miRcode, miRDB, TargetScanHuman, and miRTarBase, we identified 48 common miRNAs that were targeted by both 20 differentially expressed lncRNAs and 189 mRNAs. In accordance with these data, we constructed an lncRNA-miRNA-mRNA competitive regulation network (Fig. 5). The top 10 lncRNAs with the highest connectivity are listed (Table 1).

\section{Construction of PPI network and GO/KEGG analysis of the mRNAs interacting with IncRNAs in the ceRNA network}

To better identify critical genes and understand the biological function of mRNAs in the ceRNA network, we established a protein-protein interaction (PPI) network in STRING software [31] with interaction scores of $>0.4$ (Fig. 6) and performed GO enrichment analysis using the background of a human whole-genome (Tables 2,3). As the PPI network showed, the primary hub nodes were JUN, CXCR4, BCL6, CTGF, NOS3, VEGFC, ITPKB, PTGS2, RAC3, and KAT2B. Through an enrichment analysis of their protein interacting partners, we observed that the most significant biological processes defined by GO were the response to stimulus (GO:0048583), regulation of signaling (GO:0023051), cell communication (GO:0010646), and signal transduction (GO:0009966), and positive regulation of biological process (GO:0048518). About half of the significant molecular functions were related to transcriptional regulation (GO:0000976, GO:0000977, GO:0003700, GO00140110).

Further, KEGG enrichment analysis based on ClueGO/ CluePedia with $p<0.05$ and a kappa score $\geq 0.4$ revealed that the top five significant enrichment pathways were axon guidance, and the signaling pathways of TNF, calcium, Wnt, and NF-kappa B (Fig. 7). 


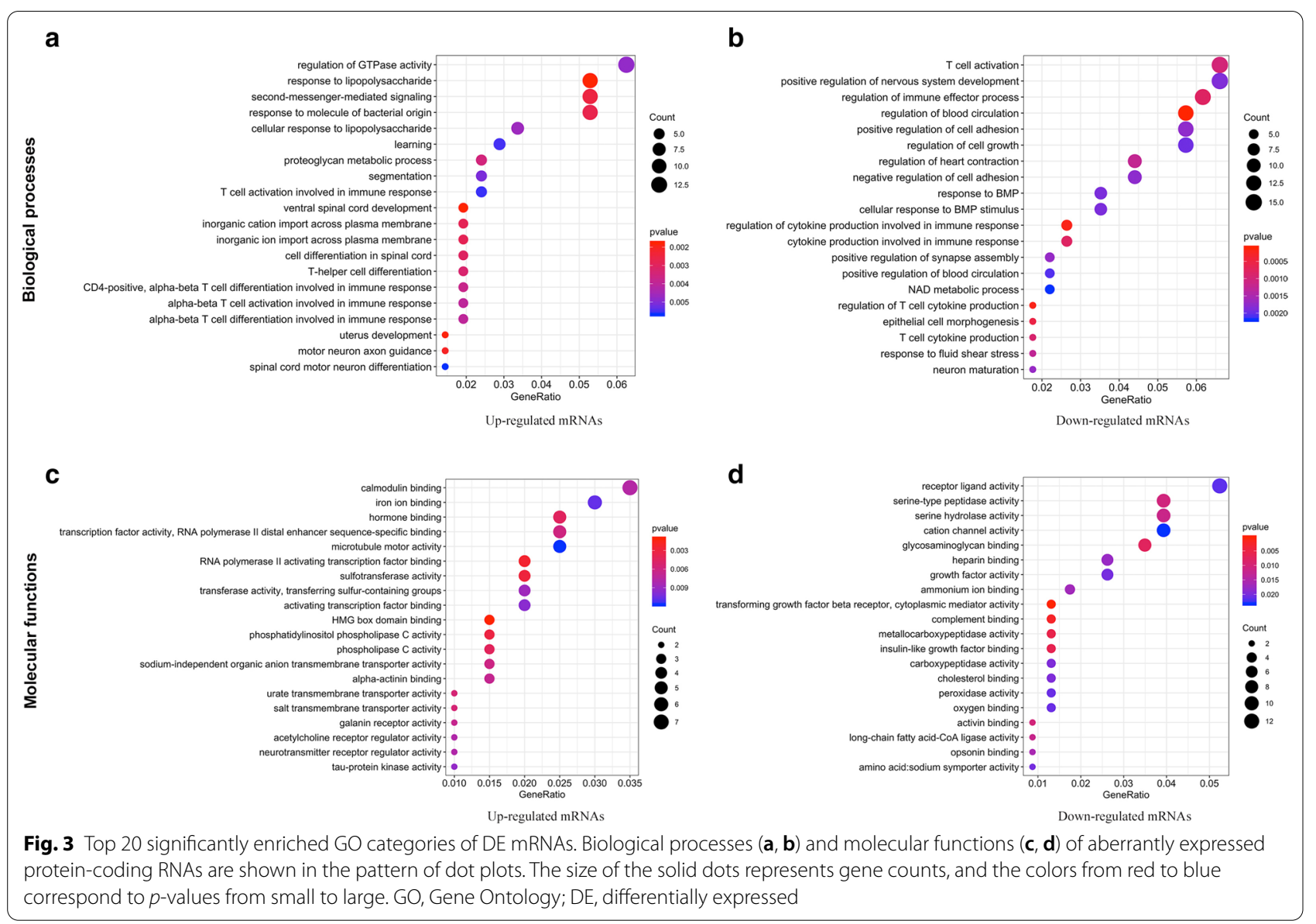

\section{Discussion}

Emerging evidence over decades has revealed that ncRNAs play a vital role in neuronal development and function, by modulating DNA replication, epigenetic modifications, controlling the transcriptional and posttranscriptional process, and by regulating miRNA activity and mRNA translation and protein stability [35-37]. Studies have shown that lncRNAs are important and poorly explored molecules related to neurological disease [38] involving Parkinson's Disease (PD), Alzheimer's Disease (AD), fragile $\mathrm{X}$ syndrome, and spinocerebellar ataxia [39-41]. Over the past few years, several lncRNAs, including HTT-AS [42], BDNF-AS [43], ABHD11AS1 [44], neural human accelerated region 1 (HAR1) [45], TUNA [46], and NEAT1 [47], among others, have been found to be related to the altered expression of HDrelated genes, and changes in these have contributed to neuronal apoptosis and the increased mHTT toxicity observed in HD. Moreover, microarray data analysis from HD brain tissues has also revealed multiple misregulated lncRNAs such as TUG1, LINC000341, RPS20P22, MEG3, DGCR5, and LINC000342, among others [17]. However, most HD-related lncRNAs with known functions mainly act as epigenetic or transcriptional regulators in the cellular nucleus; however, cytoplasmic processes remain elusive.

Studies have revealed that some lncRNAs acting as "miRNA sponges" that compete with the miRNA target gene sponge and bind MREs, which alleviates miRNAmediated target mRNA inhibition and subsequently promotes miRNA degradation through double-stranded RNA formation [48]. This ceRNA regulatory mode is one of the essential ways for ncRNAs to regulate biological functions by interacting with their downstream target mRNAs. Here, we have identified hundreds of DE lncRNAs and DE mRNAs in HD NPC cell lines, using a bioinformatics analysis method widely used in experimental and clinical studies to identify DEGs and pathways, diagnostic biomarkers, and potential therapeutic agents [49], and to determine the genetic basis of several diseases [50]. However, most of DE lncRNAs have not been investigated in previous studies. Also, we created a ceRNA regulatory network to explore their potential pathways and possible roles in HD. We found that the most 


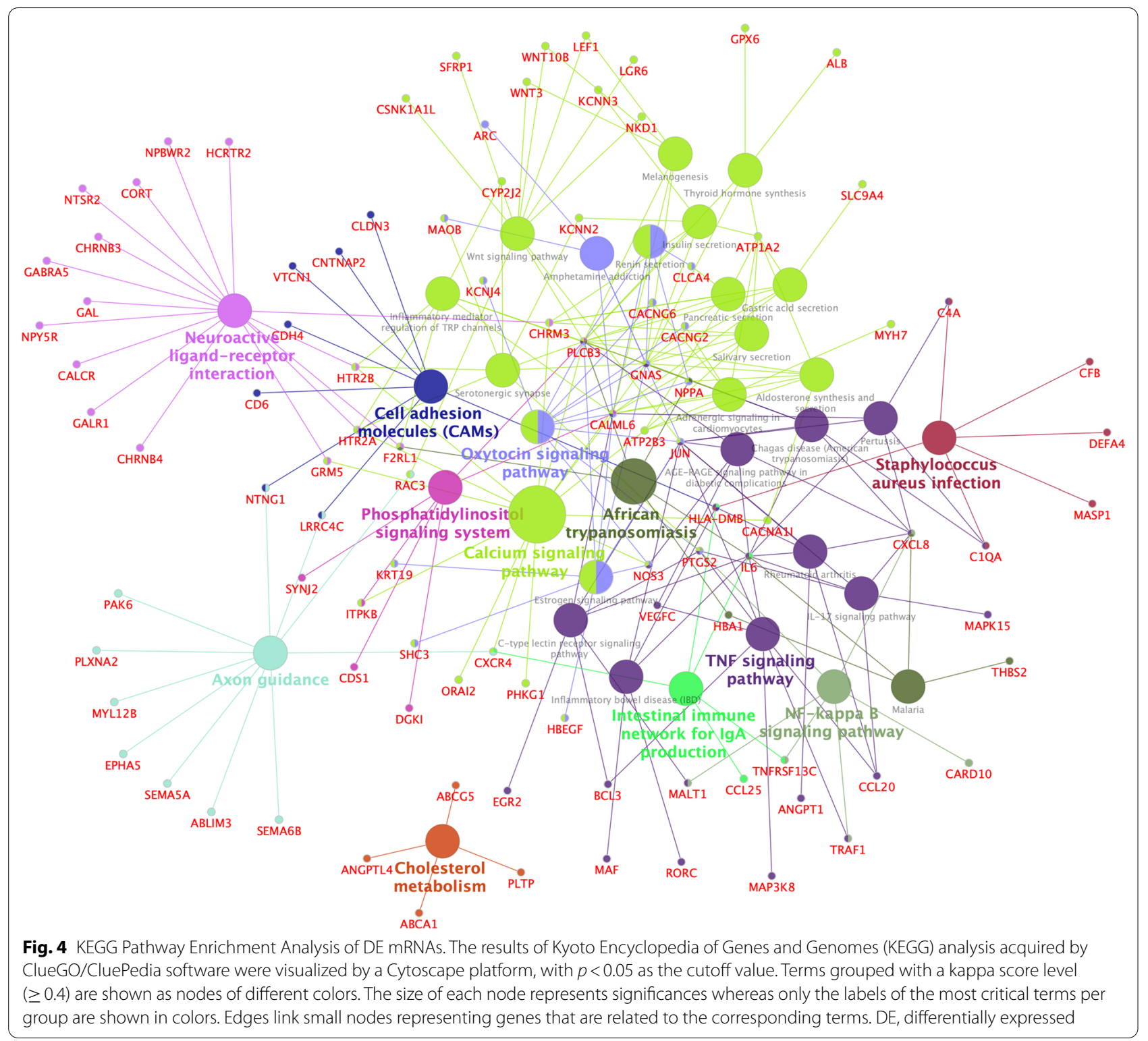

significant molecular function of mRNAs in the ceRNA network was involved in transcriptional regulation; extensive transcriptional disorder has been shown to be an early process of HD in cells and animal models, as well as in postmortem HD brain tissues $[11,51,52]$. Here, we speculate that lncRNAs in the ceRNA network may be involved in the regulation of transcription. Intriguingly, in the present study, lncRNA-HAR1B, which was previously shown to be down-regulated in HD patients, was also shown to be dysregulated in our study. It is downregulated in the striatum of patients with $\mathrm{HD}$, owing to repression by its direct target, REST, a critical neural gene regulator [45]. However, currently, little is known about the mechanism or function of HAR1B. Another significantly dysregulated lncRNA, paired box 8 (PAX8) antisense RNA1 (PAX8-AS1), is a potential regulator of PAX8 [53], which is linked to cell cycle control and metabolic processes [54]; however, its roles or mechanisms in HD remains unknown. The dysregulated lncRNA-chromosome 5 open reading frame 64 (C5orf64), of which the expression level is positively correlated with tumorinfiltrating immune cells including M2 macrophages, monocytes, eosinophils and neutrophils, can function by ceRNA network and be closely correlated with the tumor microenvironment in lung adenocarcinoma [55]. However, their functionals and roles in HD need to be further explored. 


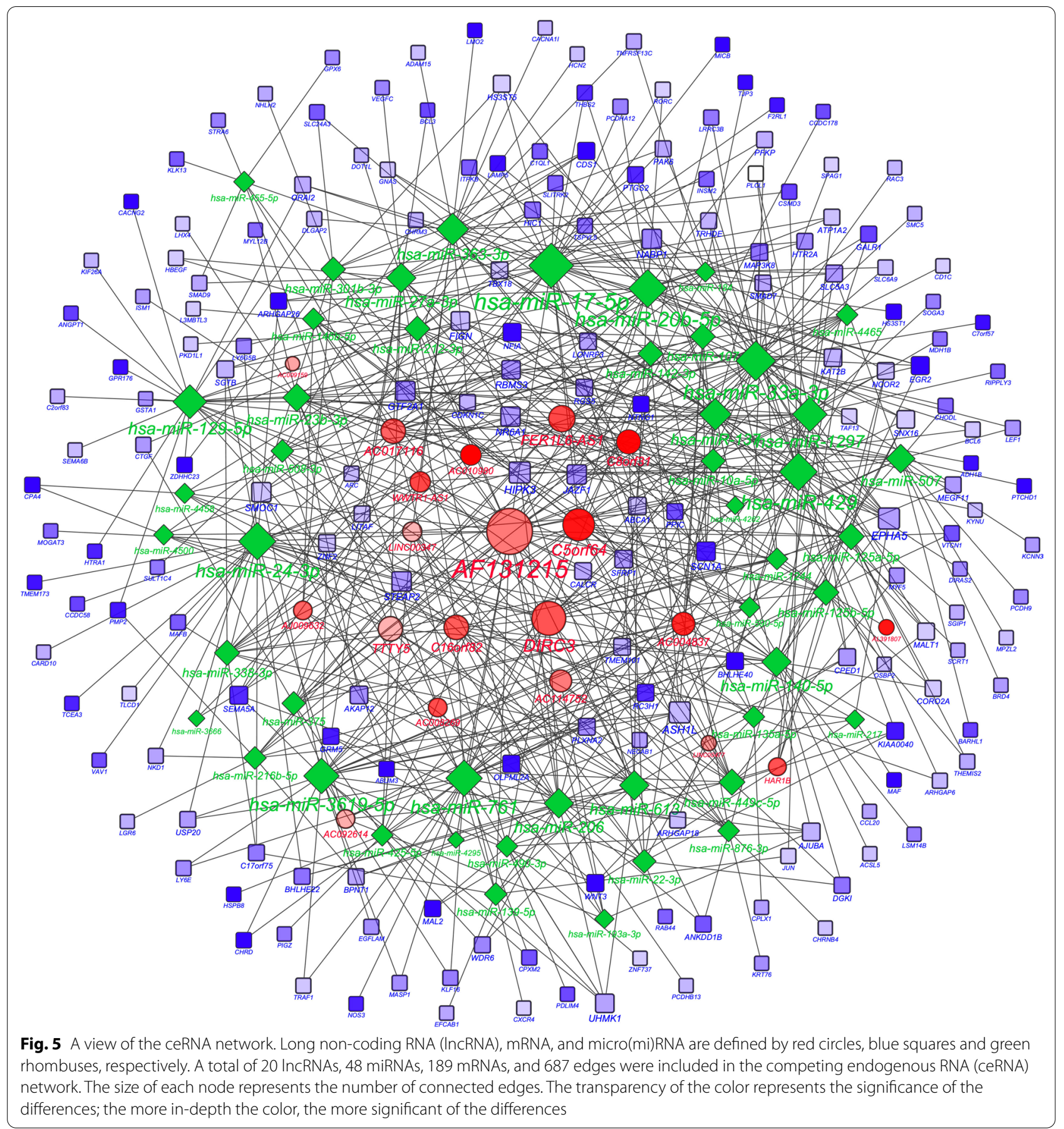

Multiple aberrant molecules observed here may contribute to the pathogenesis of HD in various ways. The transcription factor, JUN, also known as c-Jun and AP1, is markedly dysregulated in our ceRNA network and is a crucial target of the c-Jun NH2-terminal protein kinase (JNK) pathway. As an essential mediator of apoptosis in different model systems, JNK is implicated in the regulation of biological processes at both transcriptional and post-transcriptional levels [56]. JNK is activated in HD so that its inhibition may be beneficial in correcting HD-correlated neurotoxicity [57]. From our results, other transcription factors in our results, such as KAT2B, EGR2, MAFB, and GTF2A1, which may be dysregulated through ceRNA network interaction, 
Table 1 Top 10 genes with the highest connectivity in the ceRNA regulatory network

\begin{tabular}{llllll}
\hline IncRNA & Degree of connectivity & miRNAs & Degree of connectivity & mRNAs & $\begin{array}{l}\text { Degree } \\
\text { of connectivity }\end{array}$ \\
\hline AF131215 & 38 & hsa-miR-17-5p & 36 & ASH1L & 9 \\
DIRC3 & 24 & hsa-miR-33a-3p & 30 & HIPK3 & 9 \\
C50rf64 & 21 & hsa-miR-429 & 28 & EPHA5 & 9 \\
FER1L6-AS1 & 15 & hsa-miR-20b-5p & 28 & NR6A1 & 7 \\
AC017116 & 13 & hsa-miR-24-3p & 27 & STEAP2 & 7 \\
C160rf82 & 13 & hsa-miR-761 & 26 & SMOC1 & 7 \\
TTTY5 & 13 & hsa-miR-3619-5p & 25 & JAZF1 & 7 \\
C80rf31 & 12 & hsa-miR-1297 & 24 & GTF2A1 & 7 \\
AC004837 & 11 & hsa-miR-129-5p & 23 & NABP1 & 7 \\
AC114752 & 10 & hsa-miR-363-3p & 21 & FIGN & 6 \\
\hline
\end{tabular}

IncRNA long non-coding RNA, ceRNA competing endogenous RNA, miRNA microRNA

contribute to transcriptional activation and interact with JUN in the PPI network. Other molecules, for example, JUN, LGR6, SFRP1, WNT3, NKD1, RAC3 and LEF1 in the ceRNA network, might be involved in the canonical Wnt/ $\beta$-catenin signaling pathway (Fig. 7). As we know, the $\mathrm{Wnt} / \beta$-catenin signaling pathway is an important component in the development of many neurodegenerative diseases $[58,59]$. In HD models, mutHTT interferes with $\beta$-catenin degradation by binding to several components of the $\beta$-catenin degradation complex, This results in an abnormal accumulation of cytoplasmic $\beta$-catenin that cannot enter the cellular nucleus to activate the transcription of pro-survival target genes [60], leading to excessive neuronal apoptosis [61]. Therefore, further studies are needed to evaluate other molecules, as mentioned above, involved in this critical pathway in HD.

In addition to transcriptional dysregulation, neuroinflammation is also one of the typical features of most neurodegenerative diseases, including HD $[62,63]$. Previous studies have observed elevated levels of various proinflammatory cytokines in the blood and brain tissues of mice and patients with HD, suggesting that inflammation may contribute to HD progression; some inflammatory cytokines are related to the TNF signaling pathway [6466]. Moreover, the NF- $k B$ signaling pathway, by modulating the cytokine production, plays a crucial role in inflammation in HD. The overexpression of mutHTT can activate the NF- $\mathrm{kB}$ pathway by directly interacting with a critical regulator of NF- $\mathrm{kB}$, the IkappaB kinase complex; this may contribute to neurodegeneration [67]. Additionally, increased activation of NF-kB has been found in astrocytes of HD patients and in a mouse model of HD, while an inflammatory response mediated by NF- $\mathrm{kB}$ in astrocytes facilitates the pathogenesis of HD [68]. In our study, we observed how several dysregulated mRNAs, such as TRAF1, PTGS2, and MAP3K8, were involved in the inflammatory response by TNF and NF- $\mathrm{kB}$ signaling pathways (Fig. 7). This can affect a wide range of functions such as apoptosis and cell survival, as well as inflammation and immunity.

A calcium signaling pathway is critical to neuronal function. In neurodegenerative diseases, impaired $\mathrm{Ca} 2+$ signal transduction can interfere with mitochondrial function and synaptic plasticity [69]. The impairment of mitochondrial function and alteration of intracellular calcium-induced calcium release and blockade mechanisms exacerbate damage in the loop function of HD [70]. Reduced $\mathrm{Ca} 2+$ levels in the endoplasmic reticulum and enhanced store-operated calcium entry channels, which play an essential signaling function in neurons [71], lead to a synaptic decline in HD [69]. Dysregulation of GNAS, CXCR4, ORAI2, ITPKB, and CACNAII genes observed here in the calcium signaling pathway seems related to the pathogenesis of HD and needs to be further elucidated in future.

Here, we also found that many axon guidance-related genes are contained in the ceRNA network. Nevertheless, few studies have involved in the correlation between axon guidance and HD. Axon guidance is essential for intricate neural circuit formation in brain development [72]. Some studies have shown an abnormal expression or mutation of axon guidance-related genes or proteins that are indispensable for maintaining normal synaptic structure and connections in adults, and are associated with different neurological disorders [72, 73], such as $\mathrm{AD}, \mathrm{PD}$, autism spectrum disorder (ASD), amyotrophic lateral sclerosis as well as other diseases. For example, netrin-1 plays a protective role against $\mathrm{AD}$ and may be a potential target for $\mathrm{AD}[74,75]$. Mice with a deficiency in the SEMA5A gene presented with ASD-like behaviors 


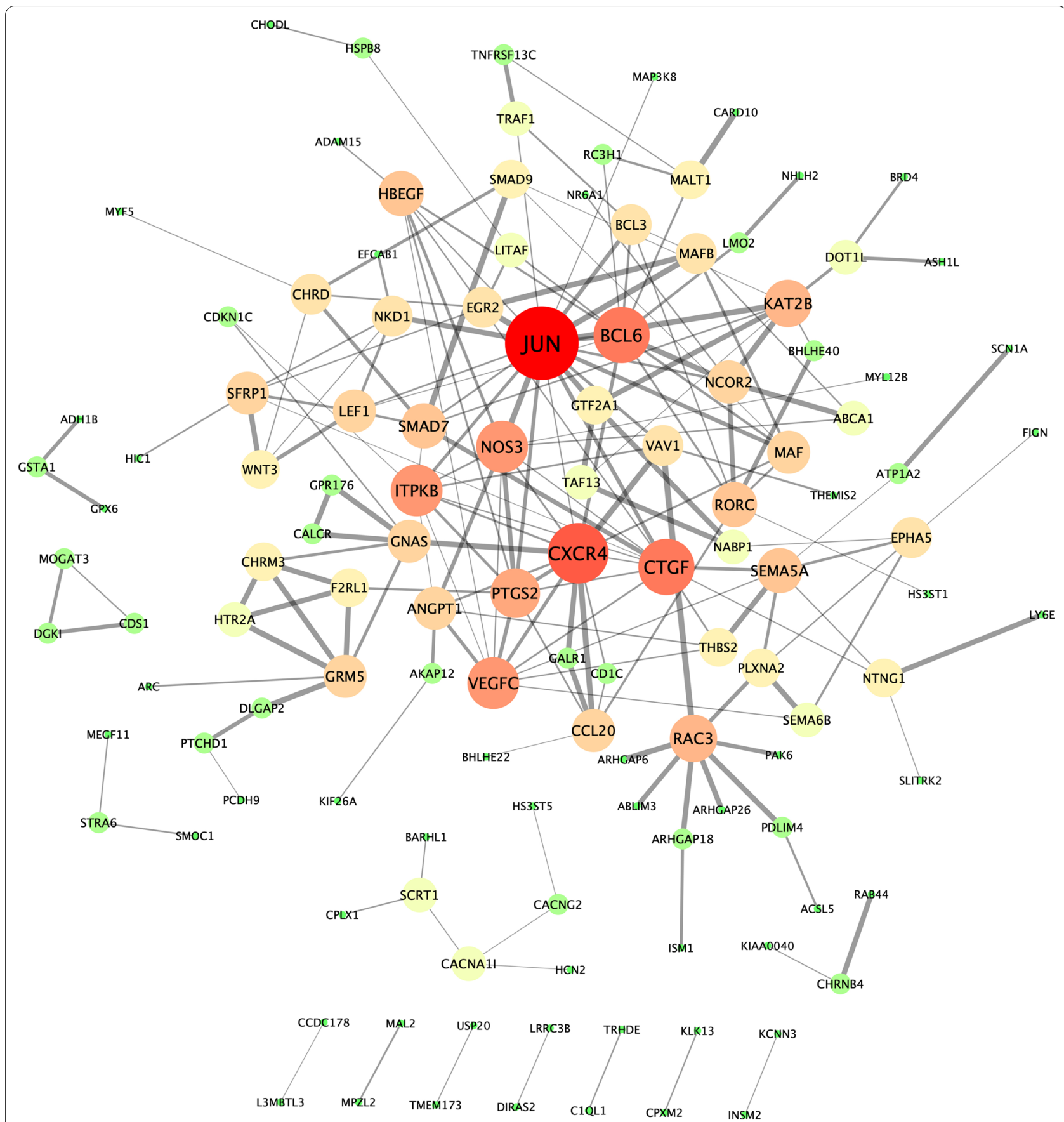

Fig. 6 The protein-protein interaction network. This protein-protein interaction (PPI) network comprises of 123 mRNAs with an interaction score $>0.4$ in STRING software that was optimized with Cytoscape software. The mRNAs are shown in different sizes and gradient colors of nodes based on the degree of edges. Green, yellow, or red correspond to low, medium, or high degrees, respectively. The edge thickness indicates interaction scores

[76]. A mutation of SEMA5A was found in a patient with ASD [77]. In HD, synaptic dysfunction is one of the typical pathogenic features, based on our results and the role of axonal guidance in other neurodegenerative diseases, and it is reasonable to believe that the aberrant axonal guidance may participate in the pathogenesis of HD. Nevertheless, relevant knowledge is limited, and further studies are needed to clarify this problem. 
Table 2 Top five terms for Biological Process (GO) of predicted mRNAs in ceRNA

\begin{tabular}{llll}
\hline GO-term & Description & Count in a gene set & False discovery rate \\
\hline GO:0048583 & Regulation of response to stimulus & $71 / 3882$ & $2.53 \mathrm{E}-05$ \\
GO:0023051 & Regulation of signaling & $65 / 3360$ & $2.53 \mathrm{E}-05$ \\
GO:0010646 & Regulation of cell communication & $65 / 3327$ & $2.53 \mathrm{E}-05$ \\
GO:0009966 & Regulation of signal transduction & $58 / 3033$ & 0.00013 \\
GO:0048518 & Positive regulation of biological process & $84 / 5459$ & 0.00070 \\
\hline
\end{tabular}

GO Gene Ontology, ceRNA competing endogenous RNA

Table 3 Top 5 terms of molecular function (GO) of predicted mRNAs in ceRNA

\begin{tabular}{llll}
\hline GO-term & Description & Count in a gene set & $\begin{array}{l}\text { False } \\
\text { discovery } \\
\text { rate }\end{array}$ \\
\hline GO:0140110 & & 0.0389 \\
GO:0044212 & Transcription regulator activity & $36 / 2069$ & 0.0389 \\
GO:0043167 & Transcription regulatory region DNA binding & $19 / 829$ & 0.0389 \\
GO:0005515 & Ion binding & $84 / 6066$ & 0.0389 \\
GO:0005488 & Protein binding & $86 / 6605$ & 0.0389 \\
\hline
\end{tabular}

GO gene ontology, ceRNA competing endogenous RNA

While the functions and mechanisms of most DE lncRNAs here remain unclear, they may play a role in the pathogeneses of HD by controlling the expression of the above-mentioned coding genes in the ceRNA network. In future, further in vitro and in vivo experiments are needed to verify the ceRAN regulatory network established here and to elucidate its roles in HD pathogenesis.

The limitations of this study should also be mentioned. First of all, we must be clear that the differentially expressed molecules screened using various bioinformatics methods only represent a certain disease stage, while changes in gene expression is an intricate and dynamic process rather than existing in a stationary system. Second, lncRNAs can play a role in different ways by virtue of their nuclear or cytoplasmic localization. Here, we only explored the role of lncRNAs as miRNA sponges in the ceRNA network in NPC cells derived from iPSC cell lines. Third, only 12 samples were used in this study, with a comparison made between a CAG33 healthy controls and CAG180 HD cell lines. In addition, as with the results acquired through various bioinformatics methods, the DE lncRNAs we obtained here need to be further validated in HD cells or animal models, including their potential expression changes and functions. Finally, the upstream regulatory molecules of lncRNAs have not been explored, and the relationship between mutHTT and the lncRNAs within this study remains to be elucidated.

\section{Conclusions}

In conclusion, by using bioinformatics methods in the present study, we have successfully identified hundreds of DE mRNAs and lncRNAs that were not previously linked to HD. By creating a ceRNA network with differentially expressed coding genes and lncRNAs, and in predicting miRNAs, it was revealed that lncRNAs may be involved in facilitating the expression of corresponding mRNAs through an lncRNA-miRNAmRNA regulatory mechanism, contributing to the pathological processes of HD. Functional and PPI network analyses demonstrated that a variety of transcription factors were dysregulated, the expression of which were controlled by lncRNAs. It is further suggested that the function of lncRNAs may be related to a wide range of transcriptional regulation. This study provides new clues to uncovering the mechanisms of lncRNAs in HD and can be used as the focus for further investigation. 


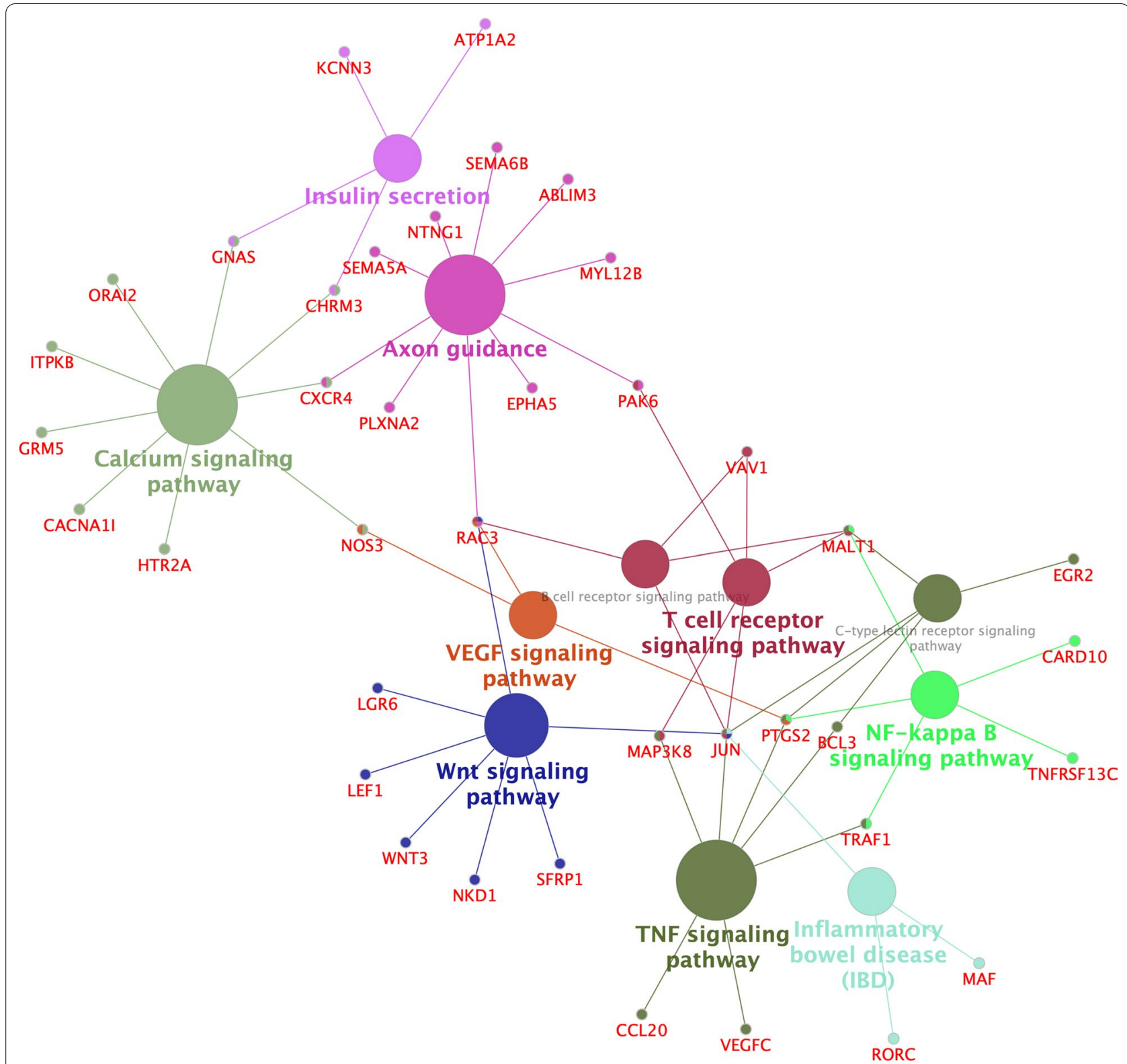

Fig. 7 A view of KEGG Pathway Enrichment Analysis of mRNAs in ceRNA. The Kyoto Encyclopedia of Genes and Genomes (KEGG) pathway enrichment graph was visualized by Cytoscape software based on ClueGO/CluePedia KEGG analysis with $p<0.05$ and a kappa score $\geq 0.4$. See Fig. 3 for the meaning of nodes and edges. ceRNA, competing endogenous RNA; VEGF, vascular endothelial growth factor; TNF, tumor necrosis factor

\section{Abbreviations}

HD: Huntington's disease; BP(s): Biological processe(s); CAG: Cytosine-adenine-guanine; ceRNA: Competing endogenous RNA; DE/DEG(s): Differentially expressed/differentially expressed gene(s); GO: Gene Ontology; HTT: Huntingtin; iPSC(s): Induced pluripotent stem cell(s); KEGG: Kyoto Encyclopedia of Genes and Genomes; IncRNA(s): Long non-coding RNA(s); MF: Molecular function; mutHTT: Mutant huntingtin; NPC(s): Neural progenitor cell(s); polyQ: Polyglutamine; PPI: Protein-protein interaction; REST: Repressor element 1 (RE1) silencing transcription factor; RISC: Repressive protein complex.

\section{Acknowledgements}

The authors are grateful to Xiaohong Xu, Yilin Tay, Bernice Sim et al. for providing the original expression dataset GSE93767 on GEO.

\section{Authors' contributions}

SC conceived and designed the study; YL, XT contributed to data analyses and created the diagrams; XT, TZ drafted and revised the manuscript. All authors reviewed the manuscript, made critical revisions and approved the final draft. All authors read and approved the final manuscript.

\section{Funding}

This study was supported by the National Natural Science Foundation of China (Grant No 81371271) and was also sponsored by the "Liaoning BaiQianWan Talents Program" and 345 Talent Project of Shengjing Hospital of China Medical University. The funders had no role in the study design and collection, analysis, and interpretation of data and in writing the manuscript. 


\section{Availability of data and materials}

The datasets used and/or analysed during the current study are available from NCBI Gene Expression Omnibus (GEO: GPL10558, GSE93767) https://www. ncbi.nlm.nih.gov/geo/

\section{Ethics approval and consent to participate}

Not applicable.

\section{Consent for publication}

Not applicable.

\section{Competing interests}

The authors declare that they have no competing interests.

Received: 9 August 2020 Accepted: 4 February 2021

Published online: 12 February 2021

\section{References}

1. MacDonald ME, Ambrose CM, Duyao MP, Myers RH, Lin C, Srinidhi L, Barnes G, Taylor SA. A novel gene containing a trinucleotide repeat that is expanded and unstable on Huntington's disease chromosomes. The Huntington's Disease Collaborative Research Group. Cell. 1993:72(6):971-83.

2. Chanda K, Das S, Chakraborty J, Bucha S, Maitra A, Chatterjee R, Mukhopadhyay D, Bhattacharyya NP. Altered levels of long NcRNAs Meg3 and Neat1 in cell and animal models of Huntington's disease. RNA Biol. 2018;15(10):1348-63.

3. Stine OC, Pleasant N, Franz ML, Abbott MH, Folstein SE, Ross CA. Correlation between the onset age of Huntington's disease and length of the trinucleotide repeat in IT-15. Hum Mol Genet. 1993;2(10):1547-9.

4. Leavitt BR, van Raamsdonk JM, Shehadeh J, Fernandes H, Murphy Z, Graham RK, Wellington CL, Raymond LA, Hayden MR. Wild-type huntingtin protects neurons from excitotoxicity. J Neurochem. 2006;96(4):1121-9.

5. Zuccato C, Ciammola A, Rigamonti D, Leavitt BR, Goffredo D, Conti L, MacDonald ME, Friedlander RM, Silani V, Hayden MR, et al. Loss of huntingtin-mediated BDNF gene transcription in Huntington's disease. Science. 2001;293(5529):493-8.

6. DiFiglia M, Sapp E, Chase KO, Davies SW, Bates GP, Vonsattel JP, Aronin N. Aggregation of huntingtin in neuronal intranuclear inclusions and dystrophic neurites in brain. Science. 1997;277(5334):1990-3.

7. Panov AV, Gutekunst CA, Leavitt BR, Hayden MR, Burke JR, Strittmatter WJ, Greenamyre JT. Early mitochondrial calcium defects in Huntington's disease are a direct effect of polyglutamines. Nat Neurosci. 2002;5(8):731-6.

8. Trushina E, Dyer RB, Badger JD 2nd, Ure D, Eide L, Tran DD, Vrieze BT, Legendre-Guillemin V, McPherson PS, Mandavilli BS, et al. Mutant huntingtin impairs axonal trafficking in mammalian neurons in vivo and in vitro. Mol Cell Biol. 2004;24(18):8195-209.

9. Orr AL, Li S, Wang CE, Li H, Wang J, Rong J, Xu X, Mastroberardino PG, Greenamyre JT, Li XJ. N-terminal mutant huntingtin associates with mitochondria and impairs mitochondrial trafficking. J Neurosci. 2008;28(11):2783-92.

10. Kuhn A, Goldstein DR, Hodges A, Strand AD, Sengstag T, Kooperberg C, Becanovic K, Pouladi MA, Sathasivam K, Cha JH, et al. Mutant huntingtin's effects on striatal gene expression in mice recapitulate changes observed in human Huntington's disease brain and do not differ with mutant huntingtin length or wild-type huntingtin dosage. Hum Mol Genet. 2007;16(15):1845-61.

11. Hodges A, Strand AD, Aragaki AK, Kuhn A, Sengstag T, Hughes G, Elliston LA, Hartog C, Goldstein DR, Thu D, et al. Regional and cellular gene expression changes in human Huntington's disease brain. Hum Mol Genet. 2006;15(6):965-77.

12. Johnson R, Buckley NJ. Gene dysregulation in Huntington's disease: REST, microRNAs and beyond. Neuromolecular Med. 2009;11(3):183-99.

13. Wayman GA, Davare M, Ando H, Fortin D, Varlamova O, Cheng HY, Marks D, Obrietan K, Soderling TR, Goodman RH, et al. An activity-regulated microRNA controls dendritic plasticity by down-regulating p250GAP. Proc Natl Acad Sci U S A. 2008;105(26):9093-8.
14. Packer AN, Xing Y, Harper SQ, Jones L, Davidson BL. The bifunctional microRNA miR-9/miR-9* regulates REST and COREST and is downregulated in Huntington's disease. J Neurosci. 2008:28(53):14341-6.

15. Yoo AS, Sun AX, Li L, Shcheglovitov A, Portmann T, Li Y, Lee-Messer C, Dolmetsch RE, Tsien RW, Crabtree GR. MicroRNA-mediated conversion of human fibroblasts to neurons. Nature. 2011;476(7359):228-31.

16. Guttman M, Amit I, Garber M, French C, Lin MF, Feldser D, Huarte M, Zuk O, Carey BW, Cassady JP, et al. Chromatin signature reveals over a thousand highly conserved large non-coding RNAs in mammals. Nature. 2009:458(7235):223-7.

17. Johnson R. Long non-coding RNAs in Huntington's disease neurodegeneration Neurobiol Dis. 2012:46(2):245-54.

18. Khalil AM, Guttman M, Huarte M, Garber M, Raj A, Rivea Morales D, Thomas K, Presser A, Bernstein BE, van Oudenaarden A, et al. Many human large intergenic noncoding RNAs associate with chromatinmodifying complexes and affect gene expression. Proc Natl Acad Sci U S A. 2009;106(28):11667-72.

19. Tsai MC, Manor O, Wan Y, Mosammaparast N, Wang JK, Lan F, Shi Y, Segal E, Chang HY. Long noncoding RNA as modular scaffold of histone modification complexes. Science. 2010:329(5992):689-93.

20. Bartel DP. MicroRNAs: target recognition and regulatory functions. Cell. 2009;136(2):215-33.

21. Salmena L, Poliseno L, Tay Y, Kats L, Pandolfi PP. A ceRNA hypothesis: the Rosetta Stone of a hidden RNA language? Cell. 2011;146(3):353-8.

22. Xu X, Tay Y, Sim B, Yoon SI, Huang Y, Ooi J, Utami KH, Ziaei A, Ng B, Radulescu C, et al. Reversal of phenotypic abnormalities by CRISPR/ Cas9-mediated gene correction in huntington disease patient-derived induced pluripotent stem cells. Stem Cell Reports. 2017;8(3):619-33.

23. Smyth GK, Speed T. Normalization of cDNA microarray data. Methods. 2003;31(4):265-73.

24. Wettenhall JM, Smyth GK. limmaGUl: a graphical user interface for linear modeling of microarray data. Bioinformatics. 2004;20(18):3705-6.

25. Li JH, Liu S, Zhou H, Qu LH, Yang JH. starBase v2.0: decoding miRNAceRNA, miRNA-ncRNA and protein-RNA interaction networks from largescale CLIP-Seq data. Nucleic Acids Res. 2014;42(Database issue):D92-97.

26. Jeggari A, Marks DS, Larsson E. miRcode: a map of putative microRNA target sites in the long non-coding transcriptome. Bioinformatics. 2012:28(15):2062-3.

27. Wong N, Wang X. miRDB: an online resource for microRNA target prediction and functional annotations. Nucleic Acids Res. 2015;43(Database issue):D146-152.

28. Agarwal V, Bell GW, Nam JW, Bartel DP. Predicting effective microRNA target sites in mammalian mRNAs. Elife. 2015;4:e05005.

29. Chou CH, Shrestha S, Yang CD, Chang NW, Lin YL, Liao KW, Huang WC, Sun TH, Tu SJ, Lee WH, et al. miRTarBase update 2018: a resource for experimentally validated microRNA-target interactions. Nucleic Acids Res. 2018:46(D1):D296-302.

30. Shannon P, Markiel A, Ozier O, Baliga NS, Wang JT, Ramage D, Amin N, Schwikowski B, Ideker T. Cytoscape: a software environment for integrated models of biomolecular interaction networks. Genome Res. 2003;13(11):2498-504.

31. Szklarczyk D, Gable AL, Lyon D, Junge A, Wyder S, Huerta-Cepas J, Simonovic M, Doncheva NT, Morris JH, Bork P, et al. STRING v11: protein-protein association networks with increased coverage, supporting functional discovery in genome-wide experimental datasets. Nucleic Acids Res. 2019:47(D1):D607-13.

32. Yu G, Wang LG, Han Y, He QY. clusterProfiler: an R package for comparing biological themes among gene clusters. OMICS. 2012;16(5):284-7.

33. Bindea G, Mlecnik B, Hackl H, Charoentong P, Tosolini M, Kirilovsky A, Fridman WH, Pages F, Trajanoski Z, Galon J. ClueGO: a Cytoscape plug-in to decipher functionally grouped gene ontology and pathway annotation networks. Bioinformatics. 2009:25(8):1091-3.

34. Bindea G, Galon J, Mlecnik B. CluePedia Cytoscape plugin: pathway insights using integrated experimental and in silico data. Bioinformatics. 2013;29(5):661-3.

35. Iyengar BR, Choudhary A, Sarangdhar MA, Venkatesh KV, Gadgil CJ, Pillai B. Non-coding RNA interact to regulate neuronal development and function. Front Cell Neurosci. 2014:8:47. 
36. Riva P, Ratti A, Venturin M. The long non-coding RNAs in neurodegenerative diseases: novel mechanisms of pathogenesis. Curr Alzheimer Res. 2016;13(11):1219-31.

37. Khorkova O, Hsiao J, Wahlestedt C. Basic biology and therapeutic implications of IncRNA. Adv Drug Deliv Rev. 2015;87:15-24.

38. Mehler MF, Mattick JS. Noncoding RNAs and RNA editing in brain development, functional diversification, and neurological disease. Physiol Rev. 2007;87(3):799-823.

39. Faghihi MA, Modarresi F, Khalil AM, Wood DE, Sahagan BG, Morgan TE, Finch CE, St Laurent G 3rd, Kenny PJ, Wahlestedt C. Expression of a noncoding RNA is elevated in Alzheimer's disease and drives rapid feedforward regulation of beta-secretase. Nat Med. 2008;14(7):723-30.

40. Faghihi MA, Zhang M, Huang J, Modarresi F, Van der Brug MP, Nalls MA, Cookson MR, St-Laurent G 3rd, Wahlestedt C. Evidence for natural antisense transcript-mediated inhibition of microRNA function. Genome Biol. 2010;11(5):R56.

41. Carrieri C, Cimatti L, Biagioli M, Beugnet A, Zucchelli S, Fedele S, Pesce E, Ferrer I, Collavin L, Santoro C, et al. Long non-coding antisense RNA controls Uchl 1 translation through an embedded SINEB2 repeat. Nature. 2012:491(7424):454-7.

42. Chung DW, Rudnicki DD, Yu L, Margolis RL. A natural antisense transcript at the Huntington's disease repeat locus regulates HTT expression. Hum Mol Genet. 2011;20(17):3467-77.

43. Zuccato C, Cattaneo E. Role of brain-derived neurotrophic factor in Huntington's disease. Prog Neurobiol. 2007;81(5-6):294-330.

44. Francelle L, Galvan L, Gaillard MC, Petit F, Bernay B, Guillermier M, Bonvento G, Dufour N, Elalouf JM, Hantraye P et al: Striatal long noncoding RNA Abhd1 1 os is neuroprotective against an N-terminal fragment of mutant huntingtin in vivo. Neurobiol Aging 2015, 36(3):1601 e1607-1616.

45. Johnson R, Richter N, Jauch R, Gaughwin PM, Zuccato C, Cattaneo E, Stanton LW. Human accelerated region 1 noncoding RNA is repressed by REST in Huntington's disease. Physiol Genomics. 2010;41(3):269-74.

46. Lin N, Chang KY, Li Z, Gates K, Rana ZA, Dang J, Zhang D, Han T, Yang CS, Cunningham TJ, et al. An evolutionarily conserved long noncoding RNA TUNA controls pluripotency and neural lineage commitment. Mol Cell. 2014;53(6):1005-19.

47. Sunwoo JS, Lee ST, Im W, Lee M, Byun Jl, Jung KH, Park KI, Jung KY, Lee SK, Chu K, et al. Altered expression of the long noncoding RNA NEAT1 in Huntington's disease. Mol Neurobiol. 2017;54(2):1577-86.

48. Ebert MS, Sharp PA. Emerging roles for natural microRNA sponges. Curr Biol. 2010;20(19):R858-861

49. Qi X, Zhang DH, Wu N, Xiao JH, Wang X, Ma W. ceRNA in cancer: possible functions and clinical implications. J Med Genet. 2015;52(10):710-8.

50. Rafehi H, Szmulewicz DJ, Bennett MF, Sobreira NLM, Pope K, Smith KR, Gillies G, Diakumis P, Dolzhenko E, Eberle MA, et al. Bioinformatics-based identification of expanded repeats: a non-reference intronic pentamer expansion in RFC1 causes CANVAS. Am J Hum Genet. 2019;105(1):151-65.

51. Consortium HDi: Developmental alterations in Huntington's disease neural cells and pharmacological rescue in cells and mice. Nat Neurosci 2017, 20(5):648-660.

52. Valor LM. Transcription, epigenetics and ameliorative strategies in Huntington's Disease: a genome-wide perspective. Mol Neurobiol. 2015:51(1):406-23.

53. Han J, Zhou W, Jia M, Wen J, Jiang J, Shi J, Zhang K, Ma H, Liu J, Ren J, et al. Expression quantitative trait loci in long non-coding RNA PAX8-AS1 are associated with decreased risk of cervical cancer. Mol Genet Genomics. 2016;291(4):1743-8

54. Bleu M, Gaulis S, Lopes R, Sprouffske K, Apfel V, Holwerda S, Pregnolato $M$, Yildiz U, Cordo V, Dost AFM, et al. PAX8 activates metabolic genes via enhancer elements in Renal Cell Carcinoma. Nat Commun. 2019;10(1):3739

55. Pang Z, Chen X, Wang Y, Wang Y, Yan T, Wan J, Wang K, Du J. Long noncoding RNA C5orf64 is a potential indicator for tumor microenvironment and mutation pattern remodeling in lung adenocarcinoma. Genomics. 2020;113(1 Pt 1):291-304.

56. Davis RJ. Signal transduction by the JNK group of MAP kinases. Cell. 2000;103(2):239-52.

57. Perrin V, Dufour N, Raoul C, Hassig R, Brouillet E, Aebischer P, Luthi-Carter $\mathrm{R}$, Deglon N. Implication of the JNK pathway in a rat model of Huntington's disease. Exp Neurol. 2009;215(1):191-200.
58. Lecarpentier Y, Vallee A. Opposite interplay between PPAR gamma and canonical Wnt/beta-catenin pathway in amyotrophic lateral sclerosis. Front Neurol. 2016:7:100.

59. Vallee A, Lecarpentier Y, Guillevin R, Vallee JN. Effects of cannabidiol interactions with Wnt/beta-catenin pathway and PPARgamma on oxidative stress and neuroinflammation in Alzheimer's disease. Acta Biochim Biophys Sin (Shanghai). 2017;49(10):853-66.

60. Godin JD, Poizat G, Hickey MA, Maschat F, Humbert S. Mutant huntingtinimpaired degradation of beta-catenin causes neurotoxicity in Huntington's disease. EMBO J. 2010;29(14):2433-45.

61. Vallee A, Lecarpentier Y, Guillevin R, Vallee JN. Aerobic glycolysis in amyotrophic lateral sclerosis and Huntington's disease. Rev Neurosci. 2018;29(5):547-55.

62. Moller T. Neuroinflammation in Huntington's disease. J Neural Transm (Vienna). 2010;117(8):1001-8.

63. Frank-Cannon TC, Alto LT, McAlpine FE, Tansey MG. Does neuroinflammation fan the flame in neurodegenerative diseases? Mol Neurodegener. 2009:4:47.

64. Bjorkqvist M, Wild EJ, Thiele J, Silvestroni A, Andre R, Lahiri N, Raibon E, Lee RV, Benn CL, Soulet D, et al. A novel pathogenic pathway of immune activation detectable before clinical onset in Huntington's disease. J Exp Med. 2008:205(8):1869-77.

65. Crocker SF, Costain WJ, Robertson HA. DNA microarray analysis of striatal gene expression in symptomatic transgenic Huntington's mice (R6/2) reveals neuroinflammation and insulin associations. Brain Res. 2006;1088(1):176-86

66. Silvestroni A, Faull RL, Strand AD, Moller T. Distinct neuroinflammatory profile in post-mortem human Huntington's disease. NeuroReport. 2009;20(12):1098-103.

67. Khoshnan A, Ko J, Watkin EE, Paige LA, Reinhart PH, Patterson PH. Activation of the IkappaB kinase complex and nuclear factorkappaB contributes to mutant huntingtin neurotoxicity. J Neurosci. 2004;24(37):7999-8008.

68. Hsiao HY, Chen YC, Chen HM, Tu PH, Chern Y. A critical role of astrocytemediated nuclear factor-kappaB-dependent inflammation in Huntington's disease. Hum Mol Genet. 2013:22(9):1826-42.

69. Pchitskaya E, Popugaeva E, Bezprozvanny I. Calcium signaling and molecular mechanisms underlying neurodegenerative diseases. Cell Calcium. 2018;70:87-94

70. Raymond LA. Striatal synaptic dysfunction and altered calcium regulation in Huntington disease. Biochem Biophys Res Commun. 2017:483(4):1051-62

71. Majewski L, Kuznicki J. SOCE in neurons: signaling or just refilling? Biochim Biophys Acta. 2015;1853(9):1940-52.

72. Kim SW, Kim KT. Expression of genes involved in axon guidance: how much have we learned? Int J Mol Sci. 2020;21(10):3566.

73. Van Battum EY, Brignani S, Pasterkamp RJ. Axon guidance proteins in neurological disorders. Lancet Neurol. 2015;14(5):532-46.

74. Shabani M, Haghani M, Tazangi PE, Bayat M, Shid Moosavi SM, Ranjbar H. Netrin-1 improves the amyloid-beta-mediated suppression of memory and synaptic plasticity. Brain Res Bull. 2017:131:107-16.

75. Spilman PR, Corset V, Gorostiza O, Poksay KS, Galvan V, Zhang J, Rao R, Peters-Libeu C, Vincelette J, McGeehan A, et al. Netrin-1 interrupts amyloid-beta amplification, increases sAbetaPPalpha in vitro and in vivo, and improves cognition in a mouse model of Alzheimer's disease. J Alzheimers Dis. 2016:52(1):223-42.

76. Duan Y, Wang SH, Song J, Mironova Y, Ming GL, Kolodkin AL, Giger RJ. Semaphorin 5A inhibits synaptogenesis in early postnatal- and adultborn hippocampal dentate granule cells. Elife. 2014;3:e04390.

77. Mosca-Boidron AL, Gueneau L, Huguet G, Goldenberg A, Henry C, Gigot N, Pallesi-Pocachard E, Falace A, Duplomb L, Thevenon J, et al. A de novo microdeletion of SEMA5A in a boy with autism spectrum disorder and intellectual disability. Eur J Hum Genet. 2016;24(6):838-43.

\section{Publisher's Note}

Springer Nature remains neutral with regard to jurisdictional claims in published maps and institutional affiliations. 